\title{
The effect of a music program on phonological awareness in preschoolers
}

\section{Franziska Degé* and Gudrun Schwarzer}

Developmental Psychology, Justus-Liebig-University, Giessen, Germany

\section{Edited by:}

Lutz Jäncke, University of Zurich,

Switzerland

Reviewed by:

Urs Maurer, University of Zurich, Switzerland

Glenn Schellenberg, University of Toronto, Canada

*Correspondence:

Franziska Degé, Developmental Psychology, Department of Psychology,

Justus-Liebig-University, Otto-

Behaghel-Str. 10F, 35394 Giessen,

Germany.

e-mail: franziska.dege@psychol.

uni-giessen.de
The present experiment investigated the effect of a music program on phonological awareness in preschoolers. In particular, the effects of a music program and a phonological skills program on phonological awareness were compared. If language and music share basic processing mechanisms, the effect of both programs on enhancing phonological awareness should be similar. Forty-one preschoolers (22 boys) were randomly assigned to a phonological skills program, a music program, and a control group that received sports training (from which no effect was expected). Preschoolers were trained for $10 \mathrm{~min}$ on a daily basis over a period of 20 weeks. In a pretest, no differences were found between the three groups in regard to age, gender, intelligence, socioeconomic status, and phonological awareness. Children in the phonological skills group and the music group showed significant increases in phonological awareness from pre- to post-test. The children in the sports group did not show a significant increase from pre- to post-test. The enhancement of phonological awareness was basically driven by positive effects of the music program and the phonological skills program on phonological awareness for large phonological units. The data suggests that phonological awareness can be trained with a phonological skills program as well as a music program. These results can be interpreted as evidence of a shared sound category learning mechanism for language and music at preschool age.

Keywords: music, music program, music training, music and language, sound category learning, phonological awareness, phonological skills, preschoolers

\section{INTRODUCTION}

Language and music are specific to humans and share several characteristics such as the use of the auditory domain as the input path and the organization of discrete perceptual elements into structured sequences (Patel, 2003). McMullen and Saffran (2004) postulated that language and music share relevant processing mechanisms, especially in childhood. One shared mechanism in childhood is the sound category learning mechanism (McMullen and Saffran, 2004). Consequently, a relationship between language sound categories such as phonemes (phonological awareness) and musical sound categories such as notes should be evident. To test this relation, the present study applied an experimental design to investigate the effect of a music program on phonological awareness in preschoolers. Moreover, the current study compared the effect of a music program and the effect of a phonological skills program on phonological awareness. If the "shared sound category learning mechanism hypothesis" (Patel, 2008) is correct, the effects of the two programs on phonological awareness should be comparable.

Empirical evidence has supported McMullen and Saffran's (2004) idea that music and language have a common basis in the early years of development. Within the first interactions between adults and infants, adults use infant-directed forms of language and music. Infants prefer these forms of infant-directed speech and songs from an early age onward (Trainor, 1996; Masataka, 1999). Moreover, infant-directed speech is often referred to as a type of musical speech (Fernald, 1991). This is in accordance with Koelsch and Siebel's (2005) assumption that the early developing brain processes language as a type of music.

Although musical and linguistic sound systems have different organizations of pitch and timbre, both sound systems face the challenge of extracting a small number of categories that are meaningful from a flow of acoustically variable signals. Moreover, the mind must separate variation within a category, e.g., induced by variable speakers, from variation that constitutes a change of category. These challenges are likely solved by a shared mechanism (Patel, 2008). This "shared sound category learning mechanism hypothesis" predicts comparable individual differences in language and musical abilities. Accordingly, the categorical building blocks of language (e.g., phonemes) should be related to the categorical building blocks of music (e.g., pitches/notes).

This hypothesis is supported by Lamb and Gregory (1993), who revealed a relationship between pitch discrimination and phonemic awareness in 4- and 5-year-old children, suggesting a general relation between musical abilities and phonological awareness. Phonological awareness describes the ability to analyze and manipulate language on two levels. On the word level, phonological awareness refers to the ability to manipulate and analyze larger phonological units (e.g., rhyming and blending words). On the phoneme level, phonological ability refers to the ability to analyze and manipulate the individual sound units (phonemes) within a word. It has repeatedly been shown that phonological awareness 
is an important predictor of later reading ability (Pratt and Brady, 1988; Bruck, 1992). Subsequently, Anvari et al. (2002) recruited a larger sample of 4- and 5-year-olds and investigated the relation between musical aptitude and the development of phonological awareness as well as the relation between musical aptitude and the development of reading. In both age groups, musical aptitude was correlated with phonological awareness. The authors concluded that the auditory processing mechanisms necessary for musical perception were related to the auditory processing mechanism necessary for phonological awareness. This relationship was further investigated with respect to the effects of music training on phonological awareness. Bolduc (2009) compared the effect of two music programs on kindergarten children. One music program employed musical activities to increase interest in reading and writing in preschoolers with special needs, whereas the other music program was primarily designed to enhance musical abilities. The music program that focused on enhancing reading and writing was more efficient in enhancing phonological awareness than the other music program. Because there was not a control group without music training, it remains unclear whether both programs significantly enhanced the phonological awareness of the children. Additionally, it is not clear whether the advancement in phonological awareness was due to the music program. In a quasi-experiment, Gromko (2005) investigated the effect of music training on phonological awareness (phoneme segmentation fluency in particular). Children in the treatment kindergarten received music training for 4 months. Children in the control kindergarten received no treatment. Gromko (2005) revealed significantly greater gains in phoneme segmentation fluency in the treatment kindergarten children than in the control kindergarten children. However, because of the pseudo-random assignment of the preschoolers to the treatment and the control group, it is not possible to interpret the results unequivocally. Children in the treatment group may have systematically differed from children in the control group with respect to confounding variables. Indeed, Gromko (2005) reported differences in socioeconomic status between the groups. Moreover, the control group did not receive an alternative training. Therefore, the significant gain in the treatment group may simply represent an effect of extra attention (Hawthorne effect).

The aim of the present experiment was twofold. Firstly, the effect of a music program on phonological awareness was investigated. Previous studies demonstrated a relation between musical abilities as well as music training and phonological awareness. However, because of the correlational and quasi-experimental approaches of these studies, it was not possible to establish the specific causation. Secondly, the current investigation compared the effect of a music program and phonological skills program on phonological awareness to examine the adaptability of the "shared sound category learning mechanism hypothesis." If this hypothesis is correct, the effect of the music program and the phonological skills program on phonological awareness should be comparable. To our knowledge, no study has addressed this question with such an approach. To this end, an experiment was conducted that randomly assigned preschoolers to a music program, a phonological skills program, and a control group that received sports training (from which no effect was expected). All preschoolers were trained for $10 \mathrm{~min}$ on a daily basis for a period of 20 weeks. Phonological awareness was tested prior to the beginning of training (pretest) and after the completion of training (post-test). This approach allowed inferences of causation and, due to random assignment, ruled out systematic differences between the groups. Moreover, the control group, which received sports training, offered the possibility to control for the effects of retesting, maturation, and attention (i.e., Hawthorne effect). Furthermore, this approach allowed the comparison between the effect of a music program and a program that explicitly addressed phonological awareness.

\section{MATERIALS AND METHODS PARTICIPANTS}

At the beginning of the experiment, the sample consisted of 55 participants. Some children transferred to a different kindergarten during the phase of trainings, and others were not available for the post-test. Five participants were excluded from the analysis because their pretest phonological skills scores were more than 2 SD from the mean. Hence, differences in phonological skills prior to training were minimized. Overall, $75 \%(n=41)$ of the original sample was included in the analyses. The dropout rate was not significantly different between the training groups and the control group. The dropouts did not differ significantly in age, gender, socioeconomic status, or intelligence from the remaining sample. The remaining participants were 41 (19 girls) preschoolers from three different kindergartens in Giessen, Germany. At the beginning of the study, the children ranged in age from 5 to 6 years $(M=5$ years; 9 months, $\mathrm{SD}=4$ months). Participants were randomly assigned to a music program ( $n=13$, six girls), a phonological skills program ( $n=14$, six girls), and a control group that received sports training ( $n=14$, seven girls). All three participating kindergartens had a music group, a phonological skills group, and a control group to rule out kindergarten effects.

\section{MATERIAL \\ Programs}

Children were trained for $10 \mathrm{~min}$ on a daily basis for a period of 20 weeks. Thus, participants received a total of 100 sessions. Preschoolers were trained in groups of five to seven children. The programs were implemented by trained research assistants. Each assistant implemented every program (phonological skills, music, sports). The preschoolers were trained at the kindergarten in a quiet room that offered sufficient space for the different tasks. A typical session comprised a short welcome (small talk, attendance check) and two different tasks that were approximately 5 minutes in duration.

The music program was created by the authors. It was based on a well-established program for early music education (Nykrin et al., 2007) and contained joint singing, joint drumming, rhythmic exercises, meter execution, training of rudimentary notation skills, dancing, and playful familiarization with intervals. Typical sessions comprised, for example, the learning of a new song and a listening exercise. At first, all children listened to the song. Then, they attempted to sing along with the CD. Finally, the group sang the song without the CD. The second task involved listening to music recordings and subsequently identifying the tempo or musical instruments on the recording. Other typical sessions involved familiarization with different instruments and joint drumming. 
The children were given the opportunity to test several instruments that were scattered across the floor on little "islands" and when indicated, they switched to the next "instrument isle." Joint drumming activities involved synchronization with a given beat as well as the creation of new beats by the children that had to be learned by the other group members. Another typical session comprised dancing to certain musical themes or synchronization of particular body parts to the music. A second task was joint singing of familiar songs and drumming while singing.

The phonological skills program was a well-established program that was specifically designed to train phonological awareness (Küspert and Schneider, 2003). It contained listening exercises, rhyming exercises, phoneme recognition exercises, syllable exercises, and the introduction of the concepts "word" and "sentence." Typical early sessions contained listening tasks that involved everyday sounds and rhyming with animal names. Children closed their eyes and guessed the sounds that the trainer produced or chose words that rhymed with an animal name such as cat. Other commonly used tasks were clapping the syllables of the animal, plant, or object displayed on a picture card as well as guessing the last phoneme of a word given by the trainer. Typical sessions at the end of the trainings phase included more difficult tasks such as the identification of words in sentences.

The sports training was created by the authors and contained exercises to train balance, physical strength, endurance, coordination, fine-motor abilities, body perception, and relaxation. Typical sports sessions contained relaxation exercises and well-directed throwing. For relaxation training, children imagined that they were an air mattress losing all air, and the throwing exercises included throwing balls into boxes and buckets of different sizes. Another typical session involved playing football in atypical body positions such as walking on their hands and feet with their belly up (crayfish football) and mastering a path with closed eyes while relying on the instruction of a teammate. Tug-of-war games and balancing objects on different body parts were also typical.

\section{Measures}

Control variables such as age, gender, intelligence, and socioeconomic status as well as phonological awareness were assessed.

Using a questionnaire designed by the authors, details about parents' education was assessed as a measure of socioeconomic status. For statistical purposes, mothers' and fathers' education were coded as a dichotomous variable, with 0 for "no university education" and 1 for "university education," and a composite variable (i.e., the average of the two scores) was used in the analyses.

To measure intelligence, the culture fair test (CFT1; Weiß and Osterland, 1977), which measures fluid intelligence, was employed. The test consisted of five subtests (substitution, mazes, classification, similarities, and matrices) and was administered in groups that did not exceed eight children. The duration of test administration was $60 \mathrm{~min}$ including instructions and breaks. Age norms were used to determine the intelligence score for each participant.

Phonological awareness was assessed with the following four subtests of the Bielefelder Screening (BISC; Jansen et al., 2002): (a) detection of rhymes, (b) synthesis of phonemes into words, (c) segmentation of words into syllables, and (d) phoneme recognition in words. Each subtest consisted of two to four practice items and
10 test items. In the detection of rhymes task, children were asked whether two words rhyme or do not rhyme (e.g., Do train and rain rhyme?). The synthesis of word task requested the synthesis of the initial sound and the remaining word (e.g., mouse) into one word. Children were asked to segment words by clapping their hands in the segmentation into syllables task. The phoneme recognition task required recognition of a particular phoneme in a word (e.g., Is there a "u" in elephant?). A composite score of all of the subtest scores was calculated. In each subtest, a maximum of 10 points was possible, resulting in a maximum composite score of 40 points. In addition, the following two composite scores were calculated: a phonological awareness score for large (words) phonological units consisting of subtest (a) and (c), and a phonological awareness score for small (phonemes) phonological units consisting of subtest (b) and (d).

\section{PROCEDURE}

Prior to the pretest, the informed consent of the parents was attained and the demographic questionnaire was sent to the participants. A pretest that assessed intelligence and phonological awareness was conducted. The intelligence test was performed in groups of five to six children, whereas phonological awareness was assessed in individual sessions, and the assessments were performed on consecutive days. The pretest was followed by 20 weeks of training. Subsequent to training, the post-test was conducted. The post-test involved the individual assessment of phonological awareness. The same test that was utilized in the pretest was applied. At the end of the project, each child received a present and a certificate for participation.

\section{RESULTS \\ CONTROL VARIABLES}

Differences in gender, age, intelligence, and socioeconomic status between the music group, the phonological skills group, and the control group were controlled. The ratio of male to female was not significantly different between the three groups, $\chi^{2}(2$, $n=41)=0.14, p=0.93$. Concerning mean age, the children in all three groups were comparable, $F(2,38)=1.04, p=0.36$, see Table 1 for mean and SD. With respect to intelligence, no significant differences between the music group, the phonological skills group, and the control group were revealed, $F(2,38)=0.16, p=0.85$, see Table 1 for details.

Children in all three groups were comparable in socioeconomic status, $\chi^{2}(4, n=37)=2.68, p=0.61$, see Table 2 for details. Four participating families did not provide details about parents' education. Taken together, the analyses of the control variables indicated that the three groups can be considered as equal in gender ratio, mean age, mean IQ, and socioeconomic status.

\section{Table 1 | Mean and SD for the control variables age and intelligence.}

\begin{tabular}{llll}
\hline Control variable & Music program & $\begin{array}{l}\text { Phonological } \\
\text { skills program }\end{array}$ & $\begin{array}{l}\text { Control group } \\
M(\mathrm{SD})\end{array}$ \\
& & $M(\mathrm{SD})$ & \\
\hline
\end{tabular}

\begin{tabular}{lccc}
\hline Age (in months) & $70.46(3.9)$ & $69.14(4.2)$ & $68.21(4)$ \\
Intelligence & $113.92(12.5)$ & $111.64(10.3)$ & $113.6(11.9)$
\end{tabular}




\section{PHONOLOGICAL AWARENESS}

At the pretest, the music group, phonological skills group, and control group did not significantly differ in phonological awareness, $F(2,38)=0.16, p=0.86$. Furthermore, the compositional scores for large and small phonological units revealed no group differences (all ps >0.6).

Phonological awareness scores were entered into a 3 (group: music, phonological skills, control) $\times 2$ (condition: pretest vs. posttest) ANOVA with repeated measures on the last factor. This analysis revealed a significant main effect of condition, $F(1,38)=16.12$, $p<0.001$. Phonological awareness improved significantly from pretest to post-test. No significant main effect for group was revealed $(p>0.5)$. Furthermore, the analysis revealed a significant group $\times$ condition interaction, $F(2,38)=3.24, p=0.05$, see Figure 1. Therefore, two ANOVAs comparing each treatment group to the control group and one ANOVA comparing the two treatment groups were calculated.

Table 2 | Distribution of parents' education as a measure of socioeconomic status within the music group, the phonological skills group, and the control group.

\begin{tabular}{llll}
\hline & Music program & $\begin{array}{l}\text { Phonological } \\
\text { skills program }\end{array}$ & Control group \\
\hline No. parent & 7 & 5 & 5 \\
At least one parent & 4 & 7 & 9
\end{tabular}

No. parent $=$ no. parent holds a university degree. At least one parent $=$ at least one parent holds a university degree. Four participating families did not provide details about parents' education.
For the comparison between the phonological skills group and the control group, phonological awareness scores were entered into a 2 (group: phonological skills, control) $\times 2$ (condition: pretest vs. post-test) ANOVA with repeated measures on the last factor. This analysis revealed a significant group $\times$ condition interaction, $F(1,26)=4.84, p=0.04$. For the comparison between the music group and the control group, phonological awareness scores were entered into a 2 (group: music, control) $\times 2$ (condition: pretest vs. post-test) ANOVA with repeated measures on the last factor. This analysis also revealed a significant group $\times$ condition interaction, $F(1,25)=7.91, p=0.009$.

The comparison of the two treatment groups revealed no significant group $\times$ condition interaction, $F(1,25)=0.06, p=0.81$. Thus, in both treatment groups, the phonological awareness score improved significantly from pre- to post-test, whereas such an improvement was not found in the control group.

Subsequently, differences in phonological awareness were analyzed in greater detail. The groups' phonological awareness on the phoneme level (small phonological units) and on the word level (larger phonological units) was compared.

For the small phonological units, a 3 (group: music, phonological skills, control) $\times 2$ (condition: pretest vs. posttest) ANOVA with repeated measures on the last factor revealed a significant main effect of condition, $F(1,38)=25.39, p<0.001$. No significant interaction or main effect for group was revealed (all $p s>0.3$ ). Similarly, in all three groups, phonological awareness of small phonological units improved significantly from pretest to post-test.

For large phonological units, a 3 (group: music, phonological skills, control) $\times 2$ (condition: pretest vs. posttest) ANOVA with repeated measures on the last factor revealed a marginal significant main effect of condition, $F(1,38)=3.39, p=0.073$.

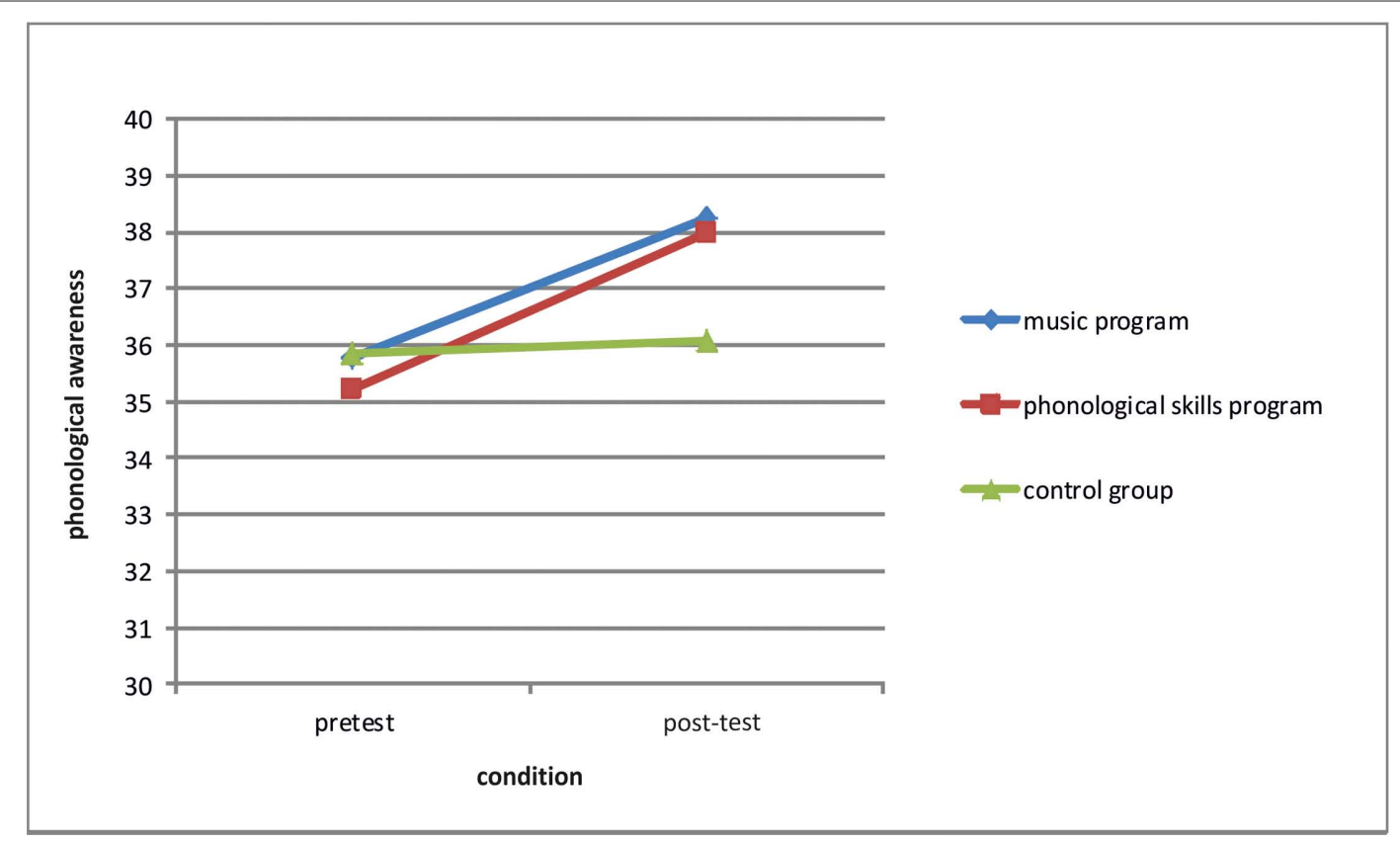

FIGURE 1 | Mean phonological awareness score at pre- and post-test in the music group, the phonological skills group, and the control group. 
No significant main effect of group was found $(p>0.7)$. Furthermore, the analysis revealed a significant group $\times$ condition interaction, $F(2,38)=4.02, p=0.026$, see Figure 2 . The individual comparison of each treatment group to the control group yielded significant interactions between group and condition. A 2 (group: phonological skills, control) $\times 2$ (condition: pretest vs. posttest) ANOVA with repeated measures on the last factor revealed a significant group $\times$ condition interaction, $F(1$, $26)=4.61, p=0.041$, for the comparison between the phonological skills group and the control group. Similarly, for the comparison between the music group and the control group, a 2 (group: music, control) $\times 2$ (condition: pretest vs. posttest) ANOVA with repeated measures on the last factor revealed a significant group $\times$ condition interaction, $F(1,25)=10.68$, $p=0.003$. Subsequent analyses $(2 \times 2$ ANOVAs with repeated measures on the last factor for the treatment groups) showed that both treatment groups significantly increased in their phonological awareness of large phonological units from pretest to post-test, $F(1,25)=7.17, p=0.009$. No significant main effect for group and no significant group $\times$ condition interaction was revealed ( $p s>0.6$ ). Thus, in both treatment groups, the phonological awareness of large phonological units score improved significantly from pre- to post-test, whereas such an improvement was not revealed in the control group.

\section{DISCUSSION}

The aim of the current experiment was to investigate the effect of a music program on phonological awareness in preschoolers. Additionally, this effect was compared to the effect of a phonological skills program, which was specifically developed to enhance phonological skills.
To this end, preschoolers were randomly assigned to a music program, a phonological skills program, and a control group that received sports training (from which no effect was expected). Participants were trained $10 \mathrm{~min}$ daily for 20 weeks. Prior to training, participants were administered a pretest on phonological awareness. Following training, the participants were retested on phonological awareness. At the pretest, no significant differences were found between the treatment groups and the control group with respect to possible confounding variables (gender, age, intelligence, and socioeconomic status). This supported the assumption that the groups did not systematically differ due to random assignment and ruled out the most likely alternative explanations of any observed trainings effect. Additionally, at the pretest, no differences in phonological awareness (overall score, small phonological units, large phonological units) were revealed. Thus, random assignment resulted in three groups that did not systematically differ on the tested variables prior to training.

A positive effect of the music program and the phonological skills program was found on phonological awareness. Preschoolers who participated in the music program or the phonological skills program significantly increased in phonological awareness, whereas such an increase was not found in the control group. Specifically, the treatment and control groups showed differential development in phonological awareness of large phonological units. Both treatment groups improved in phonological awareness of large phonological units, whereas such an improvement was not found in the control group. All three groups showed a similar development in phonological awareness of small phonological units. Thus, the experiment provides evidence of an enhancing effect of the music program on phonological awareness, in particular on phonological awareness of large phonological units.

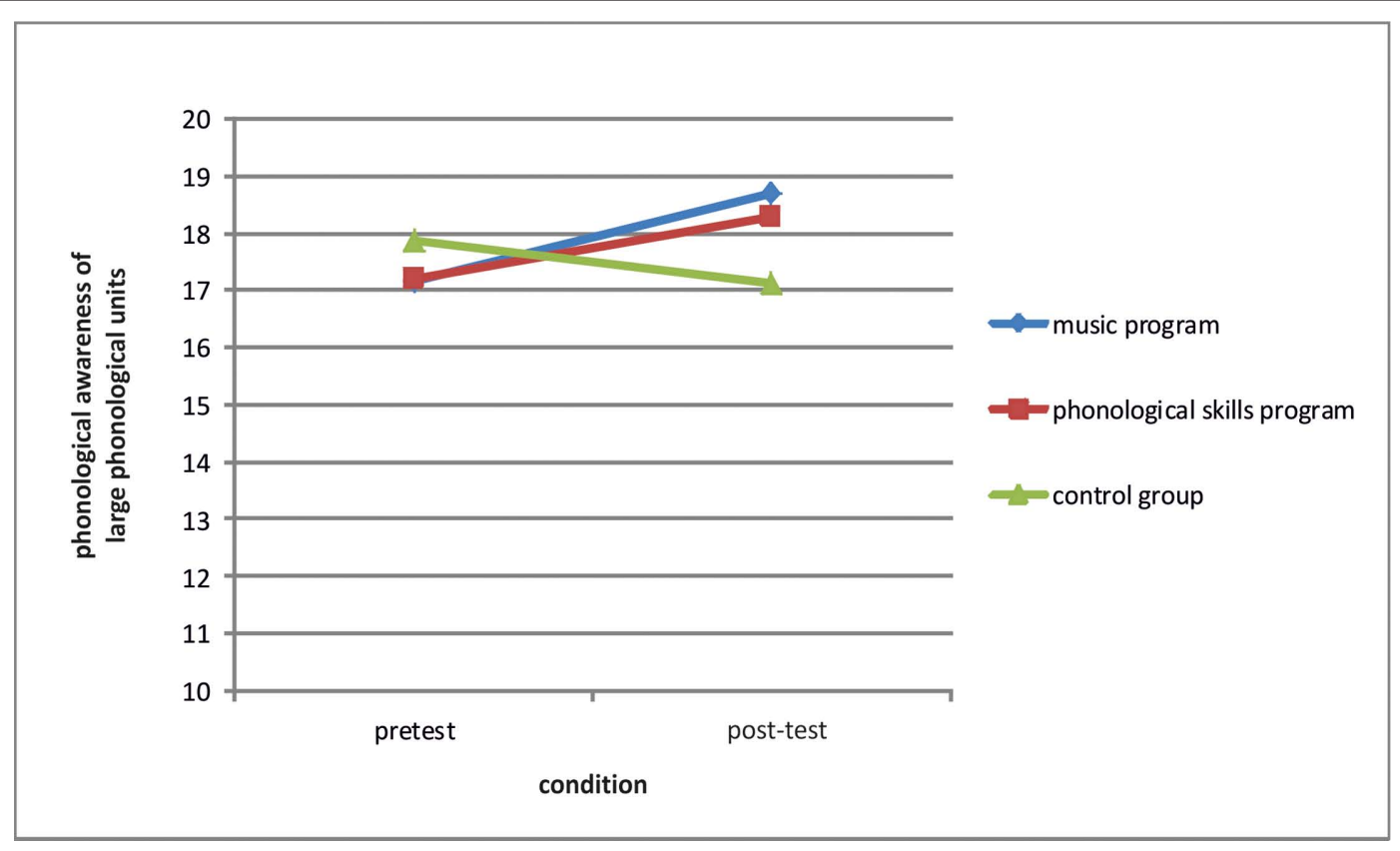

FIGURE 2 | Mean phonological awareness of large phonological units score at pre- and post-test in the music group, the phonological skills group, and the control group. 
This result is in accordance with the previous findings of Anvari et al. (2002) as well as Lamb and Gregory (1993), who demonstrated an association between musical aptitude and phonological awareness in 4- and 5-year-old children. Additionally, the present results are in line with the findings of Bolduc (2009), but emphasize the importance of a control group. In the study by Bolduc (2009), it is highly likely that both music programs increased phonological awareness. However, because there was no control group (receiving a non-musical treatment or no treatment), it is impossible to infer the degree of increase in phonological awareness caused by the less effective music program (that was designed particularly to enhance musical abilities). Moreover, the current experiment is in agreement with the results of Gromko (2005), who demonstrated an association between music training and phonological awareness. The present results extended these findings by establishing the specific causation. Because an experimental design was applied, the current results can be interpreted unequivocally in terms of an enhancing effect of the music training program on phonological awareness in preschoolers. Moreover, these results indicate that phonological awareness of large phonological units was more intensely affected by music training and phonological skills training. Thus, the overall enhancement was basically driven by the subtests that assessed phonological awareness of large phonological units. For phonological awareness of small phonological units, the treatment groups and the control group showed a similar development (an increase) over the course of the experiment. This likely represents maturation and/or effects of preschool activities in kindergartens. As preschool activities and maturation affect all preschoolers regardless of the training groups, it seems obvious that all children increased in phonological awareness of small phonological units. Additionally, the most pronounced advancement in phonological awareness of small phonological units occurs when the mapping of graphemes onto phonemes is fostered, which typically takes place in first grade (Marx, 2007). Neither program (music program or phonological skills program) focused on phoneme grapheme mapping, which might partly explain why the three groups developed similarly in phonological awareness of small phonological units. However, the phonological skills addressed small phonological units. Thus, an effect of the phonological skills program might have been expected. Nevertheless, no differences were observed between the groups in phonological awareness of small phonological units. In contrast to the similar development in phonological awareness of small phonological units, the development of large phonological units showed a differential development due to phonological skills and music training. The positive effect of the phonological skills program can be attributed to the extensive training of rhyming, segmenting, and blending. The children in this group explicitly practiced these abilities. The effects of the music training program on large phonological units represent indirect training effects. It is possible that the rhythmical exercises and the combination of rhythm and song lyrics in the children's play songs indirectly trained the ability to rhyme, segment, and blend.

The size of the positive effect of the music program $\left(d_{\text {corr }}=0.9\right)$ on phonological awareness of large phonological units was comparable to that of the phonological skills program $\left(d_{\text {corr }}=0.6\right)$ on phonological awareness large phonological units. This effect size was similar to effect sizes found in former studies of this phonological skills program (Schneider et al., 1997). The current results suggest that the music and the phonological skills programs resulted in medium to large effect sizes. Considering that the phonological skills program was explicitly designed to train phonological awareness, it is notable that the music program resulted in a similar effect size. This similarity adds evidence to the "shared sound category learning mechanism hypothesis" for language and music. During the preschool years, the mechanism for sound category learning in music and language seem to partly overlap. Thus, the proposed similarity in neuronal processing of music and language at an early age (McMullen and Saffran, 2004; Koelsch and Siebel, 2005) was supported by the present findings with respect to sound category learning. Future research should test whether the revealed influence occurs in the opposite direction. This would entail investigating effects of phonological awareness programs on pitch discrimination, for example.

A limitation of the experiment is the sample size. The sample was quite small due to the time, effort, and cost-intensive nature of the experiment as well as the initial matching. Future research should attempt to establish causation with a larger sample size. Another important limitation was that no explicit measure of motivation was employed. It is possible that children enjoyed a particular program more than another or that parents influenced children's motivation to participate. Since all activities (phonological skills program, music program, sports training) were designed for preschoolers, it is highly likely that the children enjoyed all programs equally. Nevertheless, this was not explicitly assessed. With respect to parental influence, parents were not blind to the goal (train phonological awareness) but were blind to their child's particular trainings group. However, it is possible that the child reported about the activity in the session and the parents learned their child's group placement. Nevertheless, it remains unclear whether parents practiced phonological awareness at home with the children or attempted to increase children's motivation to participate. Moreover, it is equally likely that parents of the sports training group practiced phonological awareness to prevent deficits or parents of the treatment groups to promote the child's abilities. These issues should be addressed in future research.

Considering the importance of phonological awareness in the emergence of literacy, the results point toward music programs as valuable preparation for learning to read and write. Hence, a music program could either be used as an alternative approach to train phonological awareness or used to complement existing phonological awareness programs. For a successful combination of music programs and existing phonological awareness programs, it may be important to identify the specific musical components of the music program that contributed to the advancement of phonological awareness. Then, the most important components of both training approaches could be combined.

\section{CONCLUSION}

The current results indicate that a music program can enhance phonological awareness, in particular phonological awareness of large phonological units (e.g., rhyming, segmenting, and blending). This enhancement is comparable to the effects of a phonological skills program on phonological awareness. Therefore, it seems highly likely that language and music share processing mechanisms, explicitly sound category learning mechanisms. 


\section{ACKNOWLEDGMENTS}

This research was supported by a grant for educational research (TP9 6050085) from the Justus-Liebig-University Giessen and the graduate school "NeuroAct" (neuronal rep-

\section{REFERENCES}

Anvari, S. H., Trainor, L. J., Woodside, J., and Levy, B. A. (2002). Relations among musical skills, phonological processing, and early reading ability in preschool children. J. Exp. Child Psychol. 83, 111-130.

Bolduc, J. (2009). Effects of a music programme on kindergartners' phonological awareness skills. Int. J. Music Educ. 27, 37-47.

Bruck, M. (1992). Persistence of dyslexics' phonological awareness deficits. Dev. Psychol. 28, 874-886.

Fernald, A. (1991). Prosody in speech to children: prelinguistic and linguistic functions. Ann. Child Dev. $8,43-80$.

Gromko, J. E. (2005). The effect of music instruction on phonemic awareness in beginning readers. J. Res. Music Educ. 53, 199-209.

Jansen, H., Mannhaupt, G., Marx, H., and Skowronek, H. (2002). Bielefelder Screening zur Früherkennung von
Lese-Rechtschreibschwierigkeiten. Göttingen: Hogrefe.

Koelsch, S., and Siebel, W. A. (2005). Towards a neural basis of music perception. Trends Cogn. Sci. (Regul. Ed.) 9, 578-584.

Küspert, P., and Schneider, W. (2003). Hören, lauschen, lernen Sprachspiele für Kinder im Vorschulalter, 4 Auflage. Göttingen:Vandenhoeck \& Ruprecht.

Lamb, S. J., and Gregory, A. H. (1993). The relationship between music and reading in beginning readers. Educ. Psychol. 13, 19-27.

Marx, P. (2007). Lese- und Rechtschreiberwerb. Paderborn: Schöningh UTB.

Masataka, N. (1999). Preference for infant-directed singing in 2-day-old hearing infants of deaf parents. Dev. Psychol. 35, 1001-1005.

McMullen, E., and Saffran, J. R. (2004). Music and language: a developmental comparison. Music Percept. 21, 289-311.

resentation and action control) of the University of Gießen and the University of Marburg. The authors would like to thank the participating kindergartens as well as all of the participants and their parents.

Nykrin, R., Grüner, M., and Widmer, M. (eds). (2007). Musik und Tanz für Kinder. Mainz: Schott.

Patel, A. D. (2003). Language, music, syntax and the brain. Nat. Neurosci. 6, 674-681.

Patel, A. D. (2008). Music, Language, and the Brain. Oxford: University Press.

Pratt, A. C., and Brady, S. (1988). Relation of phonological awareness to reading disability in children and adults. $J$. Educ. Psychol. 80, 319-323.

Schneider, W., Kuspert, P., Roth, E., Vise, M., and Marx, H. (1997). Short-and long-term effects of training phonological awareness in kindergarten: evidence from two German studies. J. Exp. Child. Psychol. 66, 311-340.

Trainor, L. J. (1996). Infant preferences for infant-directed versus non-infantdirected playsongs and lullabies. Infant Behav. Dev. 19, 83-92.

Weiß, R., and Osterland, J. (1977). Grundintelligenztest CFT 1 . Braunschweig: Westermann.
Conflict of Interest Statement: The authors declare that the research was conducted in the absence of any commercial or financial relationships that could be construed as a potential conflict of interest.

Received:03 March 2011; accepted: 29 May 2011; published online: 20 June 2011.

Citation: Degé F and Schwarzer $G$ (2011) The effect of a music program on phonological awareness in preschoolers. Front. Psychology 2:124. doi: 10.3389/ fpsyg.2011.00124

This article was submitted to Frontiers in Auditory Cognitive Neuroscience, a specialty of Frontiers in Psychology. Copyright (c) 2011 Degé and Schwarzer. This is an open-access article subject to a non-exclusive license between the authors and Frontiers Media SA, which permits use, distribution and reproduction in other forums, provided the original authors and source are credited and other Frontiers conditions are complied with. 